\title{
ELEMENTOS DEL SISTEMA DE PARTIDOS DE LA CATALUÑA ACTUAL
}

\author{
Joan Botella \\ (Universitat Autònoma de Barcelona)
}

\section{INTRODUCCION}

Las páginas que siguen reproducen, con pocas variaciones, el contenido de la ponencia presentada en las Jornadas «Sociedad y política, Catalunya-Euzkadi» que tuvieron lugat en Sitges (Batcelona) en septiembre de 1983. El carácter semiacadémico de esas Jornadas y la propia función de este texto, como ponencia-marco de discusión, explican sus principales limitaciones (como su orientación, básicamente descriptiva, o su insistencia en los elementos que permitieran una mayor comparabilidad entre Cata. luña y el País Vasco), así como otros aspectos menores (e.g., la ausencia de referencias bibliográficas).

Soy consciente de que el enfoque adoptado, al tomar como objeto cen. tral los partidos políticos y las relaciones que se establecen entre ellos, puede ser atacado bajo diversos ángulos, y en particular por el escaso relieve otorgado a los elementos jurídico-institucionales. No debe verse en ello una opción de principio: se trata solamente de la intuición de que esos otros aspectos (bien estudiados, por otra parte, por nuestros constitu" cionalistas), tenđrían un encaje incómodo en unas Jornadas de orientación prevalentemente sociologica. 


\section{LOS PARTIDOS PRESENTES EN CATALUNAA}

La escena política catalana ha venido presentando una imagen claramente multipartidista, con cuatto - cinco partidos relevantes. Hasta 1982, el partido ganador de las elecciones en Cataluña no ha logrado superar el 30 por 100 de los votos en ningún caso, tii han conseguido los dos primeros partidos superar la cota conjunta del 50 por 100 .

En este conjunto, la izquierda socialista y comunista ha venido conffgurando el bloque mayoritatio. Este predominio de la izquierda se debía, hasta 1982, a los resultados electorales del PSUC, ya que los resultados socialistas eran similares a los que obtenía el PSOE en el testo de España.

Por otra parte, la presencia de partidos estrictamente catalanes, sin vinculaciones orgánicas con otros partidos actuando en el conjunto de España, es importante pero modesta si se compara con la situación que se daba en el período republicano: el conjunto de estos partidos han reunido entre un mínimo del 20,2 por ciento de los votos (marzo 1979) y un máximo de1 36,5 por ciento (marzo 1980).

E1 panorama de los partidos políticos en Cataluña se ha mostrado considerablemente inestable, tanto en relación a la importancia telativa de Ias diversas fuerzas politicas (con importantes modificaciones en cada elección) como incluso en relación a la propia identidad de esas fuerzas politicas.

Ași, hay que observar que la candidatura socialista de 1977 era una coalición entre el PSC-C y la «Federación Socialista de Cataluña - PSOE»; más tarde, estos dos partidos, más el PSC-R, constituirían el actual PSC (PSC-PSOE). Por su parte, la candidatura encabezada el 1977 por Jordi Pujol, el PDC, estaba integrada por CDC, EDC, PSC-R y FNC: los dos primeros se fusionarían más tarde; el PSC-R se integró en el actual PSC, y el FNC se coaligó con ERC en 1979. En las elecciones sucesivas, CDC ha formado una coalición estable con UDC (que el año 1977 se había integrado en la UCDCC), con la denominación de CiU.

Por su parte, el partido gubernamental presentó en 1977 candidaturas integradas por independientes; en 1979, las candidaturas de CC-UCD etan coaliciones entre la organización de UCD en Cataluña y un núcleo escindido de la UDC; ambos grupos se unificarian, finalmente, en un solo partido a fines de 1979 (CC-UCD).

ERC, a su vez, aún ilegal en 1977, concurrió a las elecciones en el seno de $\mathrm{EC}$, equivalente en Cataluña de las candidaturas FDI, impulsadas en el resto de España por el PTE. Finalmente, y para limitarnos a los pattidos que han conseguido representación parlamentaria, la candidatura de AP dejó paso en 1979 a una candidatura dirigida pot el PP bajo la deno- 
Elementos del sistema de partidos de la Cataluña actual

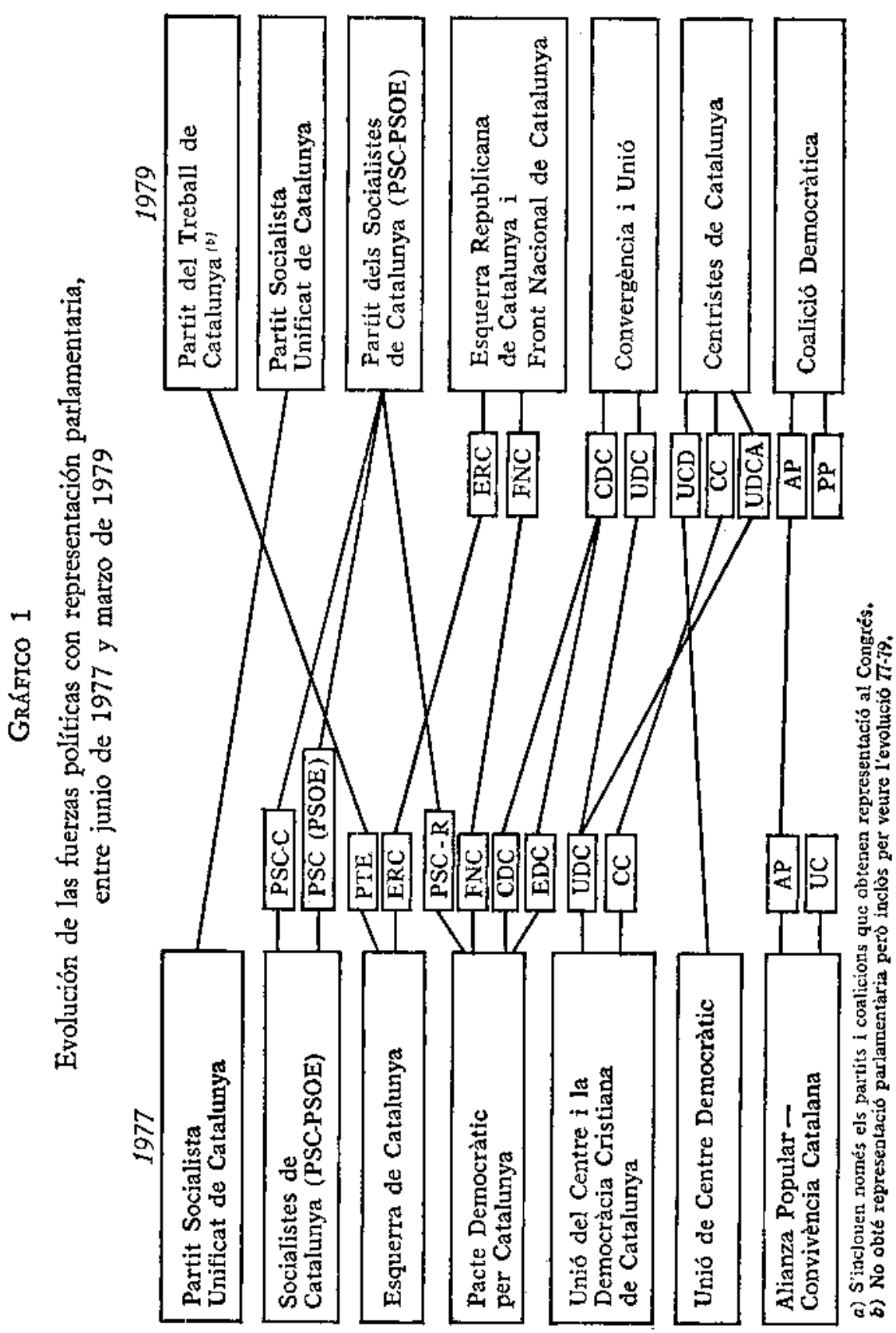


minación de $\mathrm{CD}$. Esta candidatura no concurrió a las elecciones del Parlamento de Cataluña, intentando (infructuosamente) ocupar su lugat SC.

Todos estos movimientos pueden dar la imptesión de un caos «browniano» (ver gráfico núm. 1), al que sólo escaparía el PSUC. Sin embargo, por debajo de esta aparente inestabilidad, hay unas pocas corrientes permanentes (el PSUC, vinculado al PCE; el socialismo vinculado al PSOE; los continuadores del republicanismo histórico; el «pujolismo»; el partido del gobierno español; la nostalgia del anterior régimen) que, por diversas vías y bajo diversas formulaciones, han intentado encontrat una configuración estable del espectro político catalán; este proceso ha sido más fácil en la mitad izquierda del panorama que en la zona del centro y la derecha, en la que, probablemente, aún no se ha dicho la úlitima palabra. (Véase en anexo la evolución de los resultados electroales en Cataluña.)

\section{ALGUNAS CARACTERISTICAS}

\section{DE LOS PARTIDOS CATALANES}

Si es posible intentar una caracterización general de los partidos catalanes, desde el punto de vista de sus características organizativas, habría que retener los siguientes elementos:

- Su general debilidad, en tanto que organizaciones políticas. Sólo dos partidos catalanes (PSUC y CDC) pueden pretender haber sido partidos de masas (y con un éxito limitado). La afiliación es, en getnetal, muy débil (no es pensable que hayan llegado a organizar, en conjunto, más del 2 por ciento de la población de Cataluna, incluso en el período más pletórico, en 1977); la conexión estable con organizaciones sociales no existe o se realiza con grandes dificultades, tanto para los partidos de izquierda como para los de derecha; en su financiación es completamente predominante la partida de ingresos de otigen público; etc. Así, en conjunto, los partidos catalanes no disponen de mecanismos estables de conexión cotidiana con el conjunto de la sociedad, o con aquellos sectores a los que se dirigen en primer término.

- En sentido levemente divergente, los partidos catalanes son partidos rígidos, con predominio de los organismos dirigentes sobre sus representantes públicos. Aparecen, en general, como menos personalizados que los partidos de ámbito general español (1o que, en parte, puede ponerse en relación con una cierta "succión» de figuras políticas relevantes hacia la política general española). Por último, aparecen como fuerzas claramente 
diferenciadas tanto en el plano ideológico como en el de su caracterización sociológica: no puede hablarse, en sentido estricto, de partidos «catchall», Al contrario, se ha asistido a un proceso de creciente conttaposición ideológica y social entre las diversas fuerzas políticas (proceso con consecuencias importantes sobre el funcionamiento del sistema de partidos, como se verá más abajo).

- La homogeneidad interna de los partidos catalanes es baja. Por una parte, la breve historia de la mayoría de estos partidos; por otra parte, la proyección de una dinámica de contraposición sobre una sociedad política considerablemente homogénea en el plano actitudinal; por fin, la debilidad, o inexistencia, de mecanismos de conexión eficaz con los diversos grupos sociales, han contribuido a acrecentar la conflictividad interna de los diversos partidos, que registran con frecuencia profundos debates (escasamente inteligibles pata el observador externo). La inexistencia de tendencias internas reconocidas hace que el debate se proyecte directamente a/desde los medios de comunicación (que cobran una importancia política desmesurada en el debate interno de los partidos) y favorece el surgimiento de divisiones $o$, incluso, escisiones (entre las que hay que destacar el surgimiento, a partir del PSUC, del PCC).

- Sin embargo, las características de la vida política en nuestro país, y la dinámica política «unitaria» vigente hasta 1980, han sometido a los partidos catalanes a una doble exigencia: debían mostrarse a la vez competitivos (ningún partido catalán se dirige, formalmente, a un solo sector del electorado) y cooperativos (puesto que tanto la Generalitat provisional, como la Generalitat estatutatia, como los gobietnos municipales han sido gobernados por mecanismos coalicionales, dada la ausencia de un partido clatamente mayoritario).

- Los mecanismos de articulación entre partidos catalanes y partidos del conjunto de España han adoptado diversas modalidades. En un extremo se situarían ERC y CDC, que carecen de partidos «equivalentes» en el testo de España. UDC mantiene vinculaciones leves, y probablemente indirectas, con el PNV y con núcleos democristianos del centro de España. Un paso más allá se situaría el PSUC, vinculado al PCE pero inscrito en el «Registro de Asociaciones Políticas» como partido independiente, independencia que ha defendido con una cierta energía ante intentos homogeneizadores desde el PCE. La situación del PSC es similar jurídicamente, aunque la presencia de las siglas «PSOE» en su denominación oficial sugiere una mayor vinculación que en el caso comunista. Un paso más allá se situaría CC-UCD, que, infependiente formalmente de la UCD, mantenía (incluso estatutariamente) una mayor dependencia respecto del partido de ámbito general español. Por fin, la organización de AP en Cataluña se 
configura, simplemente, como la organización regional de un partido de ámbito general español.

- Finalmente, puede afirmarse que los partidos catalanes han conseguido instaurar de un modo más completo y más eficaz el monopolio de la representación y del ejercicio del poder que los partidos del resto de España. Se ha asistido a una casi completa pérdida de importancia política de instituciones, círculos y personalidades «independientes», tal vez con mayor profundidad que en el resto de España. La especial incidencia de esta tendencia en los medios intelectuales (unida a la importancia directamente política de cuestiones como la normalización lingǘstica) podría contribuir a artojar alguna luz sobre la reciente polémica en torno a la supuesta «crisis de la cultura catalana».

\section{ALGUNOS ELEMENTOS DEL SISTEMA CATALAN DE PARTIDOS}

Como hemos advertido anteriormente, un sistema de partidos no se define solamente por la identidad de los partidos que actúan como protagonistas, sino también, y de modo muy central, por el tipo de relaciones que se establecen entre ellos; de modo más general, por el conjunto de mecanismos de interacción que se establecen.

En este sentido, Ia primera característica relevante es el número de partidos que actúan en un sistema dado. En este período, Cataluña se ha venido caracterizando por un sistema moderadamente multipartidista, en mayor medida que el sistema genetal españoi (que, incluso en el período anterio: a 1982, ha sido calificado por algunos estudiosos como bipartidista). La presencia de entre cuatro y cinco fuerzas políticas institucionalmente relevantes hace que el sistema catalán aparezca como más frag. mentado que el sistema general español: una medida usual de la fragmentación de un sistema de partidos, el índice $\left\langle\mathrm{F}_{\rangle}\right.$elaborado por $\mathrm{D}$. Rae (índice que oscila entre un valor de 0 en caso de nula fragmentación, y un valor de 1 en caso de infinita fragmentación), arroja un valor promedio para el periodo $1977-1980$ de 0,78 para Cataluña, frente a sólo 0,52 para el conjunto del sistema español. Se produce aquí una interesante inversión de tendencia respecto del período republicano, en que el sistema general español aparecía como mucho más fragmentado que el catalán (el mismo índice arroja valores promedio de, respectivamente, 0,86 y 0,63 ).

Un sistema de partióos con tal grado de fragmentación puede ges- 
tionarse, esencialmente, de dos maneras: bien estableciendo un mecanismo de coaliciones alternativas (que viene a corregir en sentido bipolar la inicial fragmentación), bien a través de mecanismos ampliamente mayoritarios (mediante la corresponsabilización de la totalidad, o de una gran mayoría, de las fuerzas políticas en la acción de gobierno). Podemos, precisamente, petiodizat la evolución reciente del sistema catalán de pattidos a partir de estos dos modelos hipotéticos: principio mayotitatio o regla de la unanimidad.

En efecto, las reglas de las relaciones entre partidos en Cataluña se han modificado sustancialmente, a pesar de que la situación, en términos estrictamente numéricos, se haya mantenido prácticamente constante. Cabe distinguir dos grandes etapas:

- En el período de la Generalitat provisional, bajo la dirección de la fuerte personalidad del presidente Tartadellas, no funcionan mecanismos de decisión mayoritaria, sino de amplia unanimidad. La constitución, a finales de 1977, de un «Consell Executiu» en el que participan todos los partidos con representación parlamentaria (excepto AP, con tuna imagen fuertemente "franquista» en aquellos momentos), con un peso más o menos proporcional a sus resultados electorales y (lo que es más sig. ficativo) con la participación de los primetos dirigentes de esos partidos políticos, evita la aparición de tendencias polarizadoras, en un doble sentido: en primer lugar, difuminando los límites entre gobieno y oposición (puesto que todas las fuerzas políticas aparecen como coresponsables); en segundo lugar, legitimando a las fuerzas formalmente extremas del espectro político: el PSUC puede participar en el gobierno sin que se tambaleen las estructuras, y la UCD puede aparecer como un partido tan catalanista como los demás.

- Sin embargo, pronto aparecerán tendencias evolutivas que apuntan hacia un marco de relaciones entre partidos ciaramente distinto.

Entre otros elementos, cabe mencionar en este sentido las elecciones legislativas de 1979 (libradas en términos de «alternativa de poder»), la ruptura de la coalición senatorial entre comunistas y socialistas (coalición que había resultado ampliamente vencedora en 1977), la constitución de los "pactos de progreso" municipales entre PSC, PSUC, $\mathrm{CiU}$ y, ocasionalmente, ERC, destinados a matginar a UCD de las áreas de poder local (acuerdos que, significativamente, no son pactos multilaterales, sino una serie de pactos bilaterales entre el PSC y cada uno de los demás partidos) y, finalmente, la ruptura del uconsenso» en un solo punto en la elaboración del anteproyecto del Estatuto de autonomía, aunque se 
trata de un punto crucial (el futuro sistema electoral de la Comunidad Autónoma) y, sobre todo, porque la ruptura se produce en términos estrictos de izquierda-derecha (PSUC y PSC frente a CiU y UCD).

- Estas tendencias a la polarización se manifestatán plenamente a partir de 1980, en torno a la elección del Pariamento autónomo, y en el período posterior.

La victoria de $\mathrm{CiU}$ en las elecciones autónomas da lugar a la constitución de un gobierno monocolor de $\mathrm{CiU}$ con apoyo parlamentario de ERC y CCUCD, mientras PSC y PSUC quedan en una situación de clara oposición (así, a pesar de haber sido invitados, ambos partidos se negarán a participar en la Comisión de transferencias a la comunidad autónoma).

Sin embargo, al ser la coalición mayoritaria una coalición sólo parlamentaria, pero no gubernamental, el gobierno de la Genetalitat se ha visto forzado a negociar permanentemente los apoyos parlamentarios necesarios, posibilitando la aparición de ptesiones bilaterales que estimulan la polarización y dificultan la estabilidad política. Así, en el período abril-diciembre de 1980, se contabilizan, en las diversas votaciones en el Parlamento de Cataluña, siete mayorías parlamentarias distintas, dos de las cuales excluían a CiU. Estas tendencias se acentúan, finalmente, por la presencia de un partido, ERC, en situación arbitral en términos de aritmética parlamentaria.

Así, en conjunto, el sistema de partidos en Cataluña se ha modificado considerablemente, pasando de una situación de multipartidismo moderado y poco competitivo (por la aplicación de mecanismos «unitarios») a una de multipartidismo aparentemente polarizado, con la exclusión «a priori» de la participación en el gobierno de determinadas fuerzas, con un gobierno minoritario sujeto a presiones bilaterales y a la aparición de mayorías cambiantes en el Parlamento. Probablemente estos elementos están en la raíz de la escasa actividad del Parlamento autónomo en los últimos meses. Ahora bien, lo que nos interesa en este momento es intentar determinar en qué medida estas tendencias evolutivas se corresponden (o no) con el estado de la opinión pública catalana. En efecto, cabría afirmar la hipótesis de que la mecánica «unitaria», consensual, vigente en la política catalana hasta 1980 , encubría una situación, entre el conjunto de la población catalana, de aguda contraposición ideológica; a la inversa, es posible la hipótesis contraria: las tendencias a la pola. rización, a la contraposición, son sólo una decisión de las «cúpulas» políticas, que no se corresponde a la realidad actitudinal de la sociedad ca. 
talana. Procede, entonces, examinar los datos disponibles respecto a las actitudes políticas de los catalanes.

\section{FRAGMENTACION Y POLARIZACION EN EL ELECTORADO CATALAN}

Como hemos indicado, el sistenna catalán de partidos aparece netamente más fragmentado que el sistema general español. Desde un punto de vista teórico, tal situación puede tener dos explicaciones: o bien la existencia de posiciones fuertemente contrapuestas en términos de izquierdaderecha, o bien la presencia de otra (u otras) línea de división política de las actitudes de los ciudadanos. Examinemos sucesivamente ambas hipótesis.

La fagmentación de un sistema de partidos puede, en primer lugar, explicarse a partir de la existencia de divisiones ideológicas profundas y bien marcadas en el seno de la sociedad: llamaremos a esa situación polarización. La existencia de un estado de polarización puede expresarse de múltiples formas y a través de diversos temas de división, pero es frecuente remitirla, en último extremo, a la bien conocida divisoria entre detechas e izquierdas, En los últimos años se ha generalizado en las encuestas de opinión el uso de escalas izquierda-derecha, tomándose la distribución de la muestra a lo largo de la escala como una estimación del grado de polarización de una sociedad. Con todas las reservas que cabe fotmulat acerca de este tipo de medidas, creemos que una escala izquierda-derecha es un instrumento de útil aproximación a la realidad, especialmente si se la sitúa en un marco comparativo. Bajo este punto de vista, la situación de la opinión catalana aparece como levemente diversa de la del conjunto de España. (Cuadro núm. 1.)

La opinión catalana aparece más inclinada a la izquierda que la del conjunto de España (las posiciones " 1 ", " 2 " y " 3 " reagrupan, respectivamente, al 45 por ciento y al 32 por ciento de la población), así como algo más dispetsa en torno a la media. Sin embargo, en ambos casos el grueso de la población se concentra en las posiciones " 2 ", " 3 " y " 4 ", es decir, izquierda, centro-izquierda y centro: 64 por ciento en Cataluña y 61 por ciento en el conjunto de España (o bien 88 por ciento y 81 por ciento, respectivamente, si excluimos los «no sabe - no contesta»). 
«Papers»: Revista de Sociologia

\section{Cuadro 1}

EI electorado catalán y español en una escala izquierda-derecha

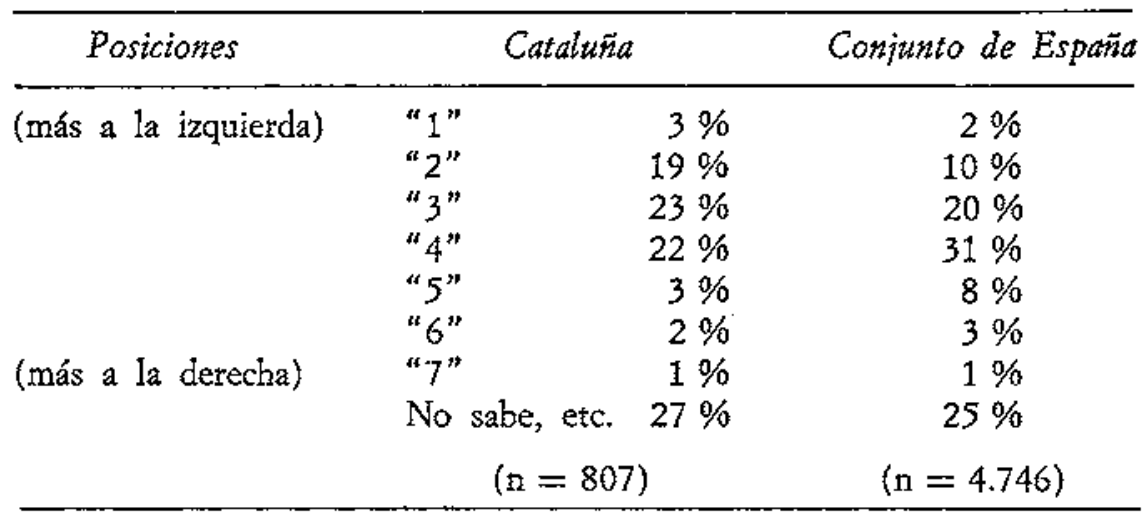

Cuadro tomado de I. Pitarch et al., Partits $i$ parlamentaris a la Catalunya d'outi (1977-1979), Barcelona, Edicions 62, 1980, p.158.

Fuente: «Encuesta sobre problemática regional», en Revista Española de Investigaciones Sociologicas, 2, 1978, 259-410.

Por consiguiente, parece que la situación catalana debe caracterizarse más bien en términos de moderación que de polarización: el grado de contraposición ideologica, en términos de izquierda-derecha, aparece como limitado ( $o$, al menos, no significativamente distinto del español).

Además, las fuerzas políticas no se dirigen a sectores específicos, como muestra la posición ideológica media de los votantes de cada partido (cuadro núm. 2), que da una imagen de mayot «apelotonamiento» hacia la zona centro-izquierda del espectro.

Por consiguiente, la distinción exclusivamente ideológica entre los electorados de los diversos partidos no constituye un criterio suficiente para distinguir entre éstos. Obsérvese, por ejemplo, como CiU y UCD, o bien PSC y ERC, se encuentran en posiciones muy poco diferenciadas.

Recapitulando: las contraposiciones ideológicas en el seno del electorado catalán, en términos de izquierda y derecha, son bajas, no pudiendo, por ellas solas, explicar la fragmentación de su sistema de partidos; además, no permiten, en un cierto número de casos, distinguir entre diversas fuerzas políticas. Es el momento, por consiguiente, de examinat la posible existencia de otras líneas divisotias, además de la de izquierda-derecha.

1. Ver nota núm. 2 . 
Elementos del sistema de partidos de la Cataluña actual

Cuadro 2

Posición ideológica media de los votantes de los partidos políticos en Cataluña

\begin{tabular}{lcc}
\hline Partido & Pasición media & Núm. de casos \\
\hline AP & 4,2 & $(17)$ \\
UCD & 4,0 & $(133)$ \\
CUU & 4,0 & $(220)$ \\
Abstención & 3,0 & $(253)$ \\
PSC & 2,9 & $(480)$ \\
ERC & 2,8 & $(43)$ \\
PSUC & 2,4 & $(242)$ \\
\hline
\end{tabular}

Naturalmente, el candidato más obvio pata integrar un posible segundo eje de división política lo constituye la cuestión de Cataluña como nacionalidad. Aunque el principio de que Cataluña constituye una nacionalidad dentro del conjunto español goza actualmente de reconocimiento constitucional y de amplio acuerdo entre las diversas fuerzas políticas, lo cierto es que a su airededor se trenzan un cierto número de cuestiones importantes y, tal vez, menos «consensuales».

Ahora bien, ¿qué cuestiones son ésas? Conviene explicitar previamente este punto, porque to es indiferente el indicador que se tome para medir las diferentes opciones existentes acerca de la cuestión nacional catalana.

Podemos, en primer lugar, concentrar nuestra atención en un dato objectivo: algo más de Ia tercera parte de la población de Cataluna ha nacido en el resto de España. Si la población de Cataluña reparte sus preferencias políticas en función de su lugar de nacimiento, tendremos aquí un elemento clave, no sólo para comprender la fragmentación de su sistema de partidos, sino para el conjunto del debate sobre la Cataluña de hoy.

Sin embargo, no parece que sea el caso. Según los datos disponibles, la distribución de los votantes de las diversas fuerzas políticas catalanas por lugar de nacimiento era la que presenta el cuadro núm. 3.2

2. Datos procedentes de una encuesta realizada en Cataluña a principios de 1980. Ver $y$. Botella y J. Matcet, «La inmigtación en Cataiuña: electores, partidos y representación políicai, en Sistema, 45, 1981: 53-73. 
«Papers»: Revista de Sociologia

\section{CuAdRo 3}

Distribución por lugar de nacimiento de los votantes de los partidos catalanes

\begin{tabular}{lcccccc}
\hline Lugar de nacimiento & UCD & CUU & ERC & PSC & PSUC & CD \\
\hline Cataluña & 66,3 & 89,6 & 88,4 & 51,4 & 47,5 & 55,6 \\
Resto de España & 33,7 & 10,4 & 11,6 & 48,4 & 52,5 & 44,4 \\
$\quad(\mathrm{n}=)$ & $(199)$ & $(231)$ & $(43)$ & $(506)$ & $(258)$ & $(9)$ \\
\hline
\end{tabular}

Tres perfiles aparecen aquí con claridad: en primer lugar, el electorado comunista y socialista, con tna mayor incidencia entre la población inmigrada (es decir, con una propotción de inmigrantes superior a la media de la población catalana); en segundo lugat, CIU y ERC, con amplio predominio de autóctonos; en tercer lugar, el perfil que ofrecía UCD, coincidente casi exactamente con la distribución general de la población de $\mathrm{Ca}$ taluña. Llama, en cambio, la atención, la inexistencia de un partido con un amplio predominio de población inmigrada entre sus votantes; el PSA, de cuyos datos no disponemos, puede haber constituido una excepción, pero sus reducidos resultados electorales muestran que no consiguió una amplia movilización del electorado inmigrante (y ello sin mencionat el hecho de que el PSA no utilizó un planteamiento «españolista/anti-catalanista», sino «andalucista», lo que es bien distinto).

Cabe, en segundo lugar, referirse a los elementos actitudinales referidos al autogobierno de Cataluña, la lengua catalana, etc. En efecto, de la inexistencia de un «cleavage» étnico, en términos de lugar de nacimiento, no se deduce forzosamente la inexistencia de un «cleavage» en el plano de las actitudes de los ciudadanos.

En este terreno, la dificultad radica en cómo medir las diversas actitudes existentes e, incluso, en determinar qué se mide. Podemos imaginar que las diferencias se producen (o se pueden producir) alrededor de tres temas distintos, tal vez coincidentes pero no idénticos:

- Un primer eje de posible diferenciación significativa puede estar constituido por los diversos grados de vivencia personal de los elementos definidores de la realidad nacional catalana. De entre ellos, el más fácilmente objetivable es el grado de conocimiento y/o utilización de la lengua catalana. Aunque no se trate de un elemento estrictamente político, es 
evidente que comporta connotaciones de gran importancia sobre el grado de integración (o de voluntad de integración) de los individuos en una comunidad.

- En segundo lugar, hay que referirse a la identidad nacional de las personas, entendida no en sentido jurídico, sino como conciencia de pertenencia a un determinado grupo nacional. En este terreno, las actitudes pueden oscilar entre un sentimiento de exclusiva pertenencia al grupo catalán y un sentimiento exclusivamente español, pasando por diversas posiciones intermedias ( $\mathrm{y}$ sin excluir que, para algunos núcleos de inmigrantes, la identidad nacional fundamental pueda dirigirse a otros territotios o comunidades).

- Finalmente, las formas de integración estatal - territorial preferidas pueden también ser diversas: podemos considerar el uniformismo centralista y el independentismo como polos extremos de un eje actitudinal que incluye, en posiciones intermedias, planteamientos descentralizadores, autonomistas, federalistas, etc.

Desgraciadamente, hay pocas estimaciones directas sobre la difusión en la población catalana ( $\mathrm{y}$ en el electotado de cada partido) de las diversas actitudes posibles en cada uno de estos ejes; además, esas estimaciones están realizadas en momentos distintos y con formulaciones tal vez no entetamente satisfactorias. Sin embargo, debemos recurrir a ellas, ya que, obviamente, Ias declaraciones programáticas de los diversos partidos pueden no coincidir con las actitudes de sus votantes.

Una encuesta de opinión realizada en 1979 planteaba a los encuestados una escala de «identidact nacional» en cinco posiciones: el cuadro número 4 presenta los resultados obtenidos. ${ }^{3}$

Los resultados que presenta el cuadro núm. 4 son sugerentes: así, si la imagen ofrecida por el electorado centrista no difiere de lo que cabía esperar «a priori», en los ottos cuatro casos hay que llamat la atención sobre aspectos poco evidentes. Así, un 20 por ciento de los votantes de ERC se consideraban «españoles» o "más españoles que catalanes» (aunque cabe tecordar que ERC era el único partido de definición republicana); sólo en el PSC el conjunto «español» y «más español que catalán» eta más nume. toso que el conjunto «catalán» y «más catalán que español»; el PSUC era el partido en que un porcentaje más alto se dectaraba simplemente «catalán, mientras que el partido en que ese grupo tomaba un valor más bajo, excluida UCD, era CiU.

3. Véase J. J. Linz et al., Informe Sociológico sobre el cambio político en España, 1975-1981. IV Informe FOESSA, Madrid (Euramérica), 1981: 548-549. 
«Papers»: Revista de Sociologia

\section{CuAdro 4}

Sentimiento de identidad nacional entre los votantes de los partidos catalanes (en \%)

\begin{tabular}{lrrrrrrrr}
\hline & PSUC & PSC & ERC & CiU & UCD & CD & Total \\
\hline $\begin{array}{l}\text { «Español» } \\
\text { «Más español }\end{array}$ & 21,3 & 29,8 & 15,4 & 8,9 & 39,1 & 38,6 & 28,8 \\
$\begin{array}{c}\text { que catalán» } \\
\text { «Tan español }\end{array}$ & 5,6 & 4,9 & 4,7 & 8,5 & 10,5 & 10,5 & 6,4 \\
$\begin{array}{c}\text { como catalán» } \\
\text { «ás catalán }\end{array}$ & 34,6 & 36,7 & 25,1 & 51,2 & 40,3 & 31,8 & 37,6 \\
$\begin{array}{c}\text { que español» } \\
\text { «atalán» }\end{array}$ & 10,2 & 10,1 & 29,1 & 28,6 & 7,5 & 9,5 & 12,3 \\
$(\mathrm{n} \Rightarrow)$ & $(104)$ & $(329)$ & $(46)$ & $(98)$ & $(218)$ & $(31)$ & $(857)$ \\
\hline
\end{tabular}

Ahora bien, lo más significativo tal vez sea la confrontación entre estas distribuciones y las que presentaba el cuadro núm. 3, acerca del lugat de nacimiento de los votantes de las distíntas fuerzas políticas: está claro que existe una considerable independencia entre el origen «territorial» de los ciudadanos y su conciencia «nacionai». Cosa distinta es que aquuí se abran interesantes pistas de análisis, al menos en dos direcciones: en primer lugar, acerca de la capacidad de los partidos políticos de transmitir su programa a sus votantes; en segundo lugar, acerca de la conflictividad potencial, en el seno de cada familia política, derivada de la disonancia entre dato «objetivo» y conciencia colectiva.

Algo similar puede decirse acerca de la otra dimensión antes mencionada, la forma de instrumentación jurídico-constitucional preferida con res" pecto a la integración estatal-territorial de España. El cuadro núm. 5 muestxa los resultados de una encuesta realizada en Cataluña acerca de ese punto (si bien debe tenerse en cuenta que la encuesta se tealizó antes de las elecciones de 1979, pot lo que se toman como criterio las principales candidaturas que habían concurtido en 1977, por lo que los datos deben tomarse «cum granu salis»; en cuanto al contenido atribuido a cada una de las cuatto posibles salidas institucionales, véase nota). ${ }^{4}$

4. Para los datos sobre Cataluña, J. J. Linz, et al., citado, pp. 546-547; para el País Vasco, p. 527. Para Ia definición de las categorías utilizadas, ver ibid., p. 515. 
Elementos del sistema de partidos de Ia Cataluña actual

CuAdro 5

Actitudes sobre la forma de autogobierno deseable, entre los votantes de 1977 (en \%)

\begin{tabular}{lrrrrrrr}
\hline & PSUC & PSC & EC & PDC & UCD & AP & Total \\
\hline Centralismo & 8 & 13 & 2 & 19 & 26 & 69 & 19,1 \\
Autonomia & 52 & 49 & 23 & 45 & 49 & 13 & 44,3 \\
Federalismo & 29 & 28 & 55 & 33 & 20 & 17 & 25,7 \\
Independencia & 11 & 8 & 21 & 4 & 3 & - & 10,9 \\
$\quad(n=)$ & $(224)$ & $(365)$ & $(28)$ & $(87)$ & $(302)$ & $(50)$ & $(1.480)$ \\
\hline
\end{tabular}

Ta1 vez el aspecto más destacado del cuadro núm. 5 sea el predominio, en general, de las posiciones «autonomista» y «federalista, que reúnen entre el 69 por ciento (UCD) y el 81 por ciento (PSUC) del electorado de cada partido; igualmente, y con la excepción de EC, la posición autono. mista es más numetosa que la federalista. Las posiciones independentistas/ anti-centralistas son más fuertes en EC y, con alguna diferencia, en PSUC. En todo caso, es visible la diferencia con la situación vasca, en que esta misma encuesta encontraba que la opción «independencia» era preferida por el 71 por ciento de los votantes de $\mathrm{HB}, 61$ por ciento de EE, 43 por ciento del PNV, y también 25 por ciento del PCE-EPK, 15 por ciento de PSOE-PSE y 5 por ciento de UCD.

En segundo lugar, y como habiamos constatado anteriormente, también en este terteno las actitudes de los ciudadanos parecen relativamente independientes de su lugar de racimiento.

Así, hay que observar que una adecuada comprensión del sistema catalán de partidos exige interpretatlo a partir del juego de dos dimensiones: la primera, constituida por un eje izquierda-derecha; y la segunda, constituida no por el hecho de la inmigración, sino por las actitudes de los ciudadanos acerca de la forma de autogobierno deseada y por su conciencia nacional. Peto lo más significativo aquí (ver gráfico núm. 2) es que esta segunda dimensión diferencia a las fuerzas políticas más que la primera y, además, las difetencia de modo desigual: su impacto es más acusado en la mitad izquierda del espectro ideológico que en su mitad derecha.

En efecto, por una parte, la diferenciación que esa segunda dimensión introduce en el conjunto CiU-UCCD-AP es menor que la introducida en el conjunto ERC-PSC-PSUC; peto es que, además, mientras que la diferencia- 
ción que introduce en la mitad derecha del espectro tiende a coincidir con los datos objetivos acerca del lugar de nacimiento de sus votantes, en la mitad izquierda esto no se da: simplificando, podríamos decir que PSC y PSUC tiener unos votantes más «catalanistas de lo que les correspondería si esta actitud dependiera, única y exclusivamente, de su lugar de nacimiento.

\section{Gráfico 2}

Representación bidimensional de la competición entre partidos en Cataluña (' $x$ ', escala izquierda-derecha; ' $y$ ', porcentaje de electores que se sienten más catalanes que españoles). Cada partido está representado por un punto, correspondiente a la situación media de su electorado en ambas escalas. La linea discontinua representa los valores medios del conjunto de Ia población de Cataluña.

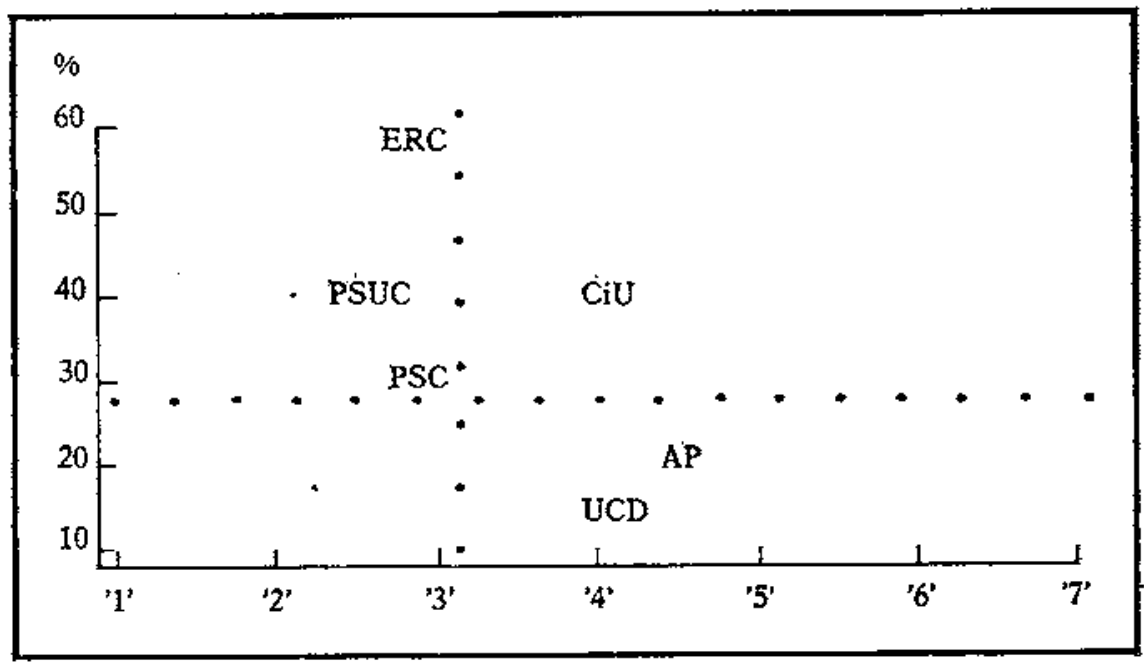

Así, la aparente proximidad entre ERC, PSC y PSUC se disuelve si tenemos presente las distintas características «objetivas» del electorado de estos tres partidos. Y ésta tal vez sea la clave de la dinámica de la competencia electoral en Cataluna: la competencia en el terreno «nacional» es más intensa en la mitad izquierda del espectro, pero no porque las posiciones de los partidos sean enormemente distintas, sino porque hay una considerable distancia $(o$, como mínimo, disonancia) entre base objetiva y actitudes ideológicas. 
Conviene, sin embargo, no llevar muy lejos este análisis: si bien es cierto que esta segunda dimensión de la política catalana existe y juega un papel indiscutible, también es cierto que la polarización a lo largo de esta dimensión es moderada; que este «cleavage» no ha sido planteado, hasta el momento, de forma directa; que no han aparecido fuerzas politicas relevantes con planteamientos anti-catalanistas; y que los partidos de izquietda con un importante componente de inmigrados son, en el plano actitudinal, $\tan$ catalanistas como el electorado de CiU.

\section{SINTESIS}

A modo de resumen los elementos que, en nuestro análisis, caracterizan de una manera más adecuada ei sistema de partidos en Cataluña en el período estudiado son los siguientes:

- En tanto que organizaciones políticas, los partidos catalanes son organizaciones débiles, desde el punto de vista de su afiliación, canales de influencia, financiación, etc.

- Sin embargo, en tanto que vehículos de la representación política de los ciudadanos, han alcanzado un predominio completo, desplazando a todo otro tipo de posibles protagonistas de la vida política.

- El sistema de partidos de Cataluña se ha caracterizado por un pluripartidismo limitado, con entre cuatro y seis partidos relevantes, y entre los cuales el matco general de relaciones ha atravesado, "grosso modo", dos grandes etapas: una primean, hasta 1979, caracterizada por la existencia de mecanismos ampliamente mayoritarios (o unitarios), y una segunda, desde 1980 , en la que la vida política se ha caractetizado por una tendencia al desartollo de mecanismos clásicos de mayoría-oposición entre centro-derecha e izquierda.

- Estos mecanismos de contraposición entre fuerzas políticas no representan la existencia de fuertes tensiones en el seno del electorado: el electorado catalán parece, en el plano actitudinal, poco propenso a la existencia de fuertes contraposiciones, aunque parece necesario tomar en cuenta, para entender adecuadamente sus comportamientos políticos, la influencia de dos «ejes» actitudinales distintos: una primera divisoria, que contrapone derecha e izquierda, y una segunda línea divisoria, trazada alrededor de las actitudes nacionales de los ciudadanos (más que del mero hecho de la inmigración). 
«Papers»: Revista de Sociologia

CuADRO 6

Evolución electoral en Cataluña, 1977-1982 (en \%)

\begin{tabular}{lcccccccc}
\hline & \multicolumn{2}{c}{1977} & \multicolumn{2}{c}{1979} & \multicolumn{2}{c}{1980} & \multicolumn{2}{c}{1982} \\
& censo & votos & censo & votos & censo & votos & censo & votos \\
\hline PSC & 22,5 & 28,4 & 20,0 & 29,2 & 13,9 & 22,3 & 36,3 & 45,2 \\
PSUC & 14,4 & 18,2 & 11,7 & 17,7 & 11,6 & 18,7 & 3,7 & 4,6 \\
PDC- & & & & & & & & \\
$\quad$ CiU & 13,3 & 16,8 & 11,0 & 16,1 & 17,2 & 27,6 & 17,8 & 22,2 \\
UCD- & & & & & & & & \\
$\quad$ CC-UCD & 13,3 & 16,8 & 13,0 & 19,0 & 6,5 & 10,6 & 1,6 & 2,0 \\
CDS & - & - & - & - & - & - & 1,5 & 1,9 \\
EC-ERC & 3,6 & 4,5 & 2,8 & 4,1 & 5,5 & 8,9 & 3,2 & 4,0 \\
AP-CD- & & & & & & & & \\
$\quad$ SC & 2,7 & 3,5 & 2,4 & 3,6 & 1,5 & 2,3 & 11,6 & 14,5 \\
PSA & - & - & - & - & 1,6 & 2,6 & $\cdots$ & - \\
UCDCC & 4,4 & 5,6 & - & - & - & - & - & - \\
\hline
\end{tabular}

En este cuadro se recogen los resuttados obtenidos en Cataluna por los partidos con representacion parlamentaria, en las elecciones gemerales de 1977, 1979, 1982, y en las elecciones autonomas de 1980 . 
Elementos del sistema de partidos de la Cataluña acfual

SIGLAS UTILIZADAS

$\begin{array}{ll}\text { AP: } & \text { Alianza Popular } \\ \text { CC-UCD: } & \text { Centristes de Catalunya-UCD } \\ \text { CD: } & \text { Coalición Democrática } \\ \text { CDC: } & \text { Convergència Democràtica de Catalunya } \\ \text { EC: } & \text { Esquerra de Catalunya } \\ \text { EDC: } & \text { Esquerra Democràtica de Catalunya } \\ \text { ERC: } & \text { Esquerta Republicana de Catalunya } \\ \text { FNC: } & \text { Front Nacional de Catalunya } \\ \text { PCC: } & \text { Partit dels Comunistes de Catalunya } \\ \text { PCE: } & \text { Partido Comunista de España } \\ \text { PDC: } & \text { Pacte Democràtic de Catalunya } \\ \text { PNV: } & \text { Partido Nacionalista Vasco } \\ \text { PP: } & \text { Partido Popular } \\ \text { PSC-C: } & \text { Partit Socialista de Catalunya (Congrés) } \\ \text { PSC-R: } & \text { Partit Socialista de Catalunya (Reagrupament) } \\ \text { PSC (PSC-PSOE): Partit dels Socialistes de Catalunya (PSC-PSOE) } \\ \text { PSOE: } & \text { Partido Socialista Obrero Español } \\ \text { PSUC: } & \text { Partit Socialista Unificat de Catalunya } \\ \text { PTE: } & \text { Partido del Trabajo de España } \\ \text { SC: } & \text { Solidaritat Catalana } \\ \text { UCD: } & \text { Unión del Centro Democtático } \\ \text { UCDCC: } & \text { Unió del Centre i la Democràcia Cristiana de Catalunya } \\ \text { UDC: } & \text { Unió Democràtica de Catalunya }\end{array}$

